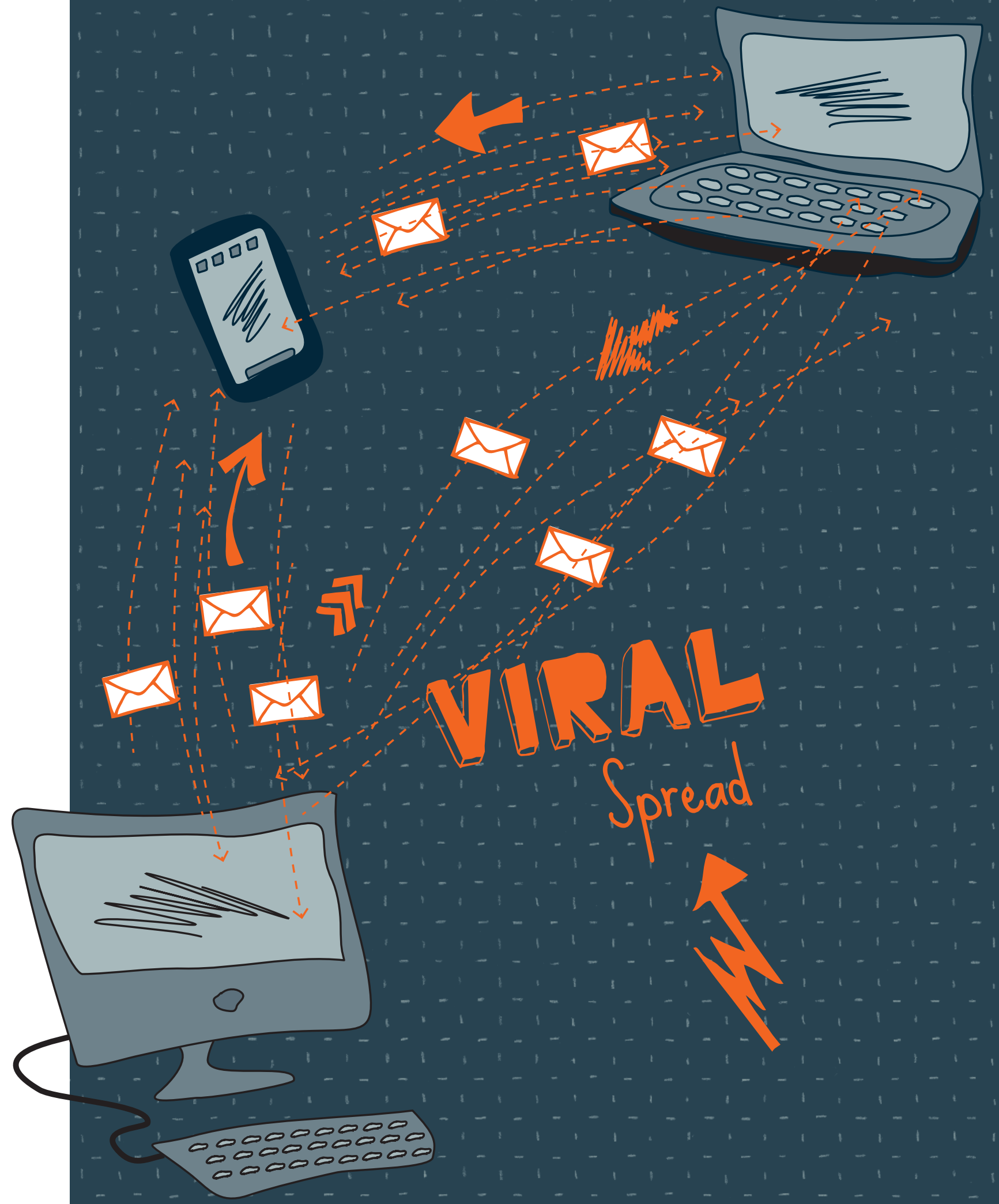




\title{
Designing Viral Product Features for Broader Reach
}

\author{
Sinan Aral and Dylan Walker
}

KEYWORDS

Viral Marketing, Peer Influence,

Social Contagion, Social Networks, Viral Product Design

THE AUTHORS

Sinan Aral,

MIT Sloan School of Management, Cambridge, Massachusetts, U.S., sinana@mit.edu

Dylan Walker,

Boston University School of Management,

Boston, Massachussetts, U.S., dtwalker@bu.edu
Companies increasingly rely on "network" and "viral" marketing within their communication strategies. The idea is that a message should spread like a biological virus and reach as many individuals as possible. While some campaigns are tremendously successful, others languish far below expectations. Among the commonly accepted factors for success are the quality of the message and the targeting of influential multipliers. This study looks at a new aspect in diffusion research: how to design a product in a way that encourages virality.

Viral product design - the process of explicitly engineering products so they are more likely to be shared among peers is not exactly new. It has existed at least since the first chain letter was sent in 1888. However, viral features have become more sophisticated, and information technology is now used to spread product awareness. This study compares how two different features of a viral product influence the spread of an application. Box 1 describes the features, and Box 2 outlines the field experiment. 
Viral product features boost diffusion /// Though the demographics and Facebook activity patterns of the users in the three groups were the same, measures of peer response to the viral features differed significantly.

> Compared with the control group, the percentage of adopters in a user's local network was approximately $450 \%$ higher for those in the passive-broadcast group and $750 \%$ higher for those in the active-personalized group that enabled both personal invitations and broadcast notifications. These figures confirm that word of mouth is considered more effective at promoting product contagion when it is personalized and active.

$>$ Measures of the speed of adoption revealed that the viral features increased the spread of the app. The time it took for the first peer adoption of the app was approximately $200 \%$ shorter for users in the passive-broadcast treatment group and approximately $300 \%$ shorter in the activepersonalized group.

> Maximal diffusion depth showed the extent to which a viral feature led to adoption beyond a user's immediate local network. The average maximal diffusion depth was approximately $360 \%$ greater for the passive-broadcast treatment group and $450 \%$ greater for the active-personalized treatment group compared with users who received neither feature.

> Finally, average app activity was approximately $130 \%$ higher in the passive-broadcast treatment group and $140 \%$ higher in the active-personalized treatment group. As more of their peers adopted the app, users were more engaged and used the app more themselves. While notifications increased short-term use, invitations had a sustained effect.
$\{$ Box 1$\}$

MOST COMMON VIRAL

PRODUCT FEATURES

While viral characteristics are fundamentally about content and the psychological effects it can have on a user's desire to share a product with peers, viral features affect how a product is shared. They enable communication and promote the product's use by other consumers. Examples of typical viral features include hypertext embedding of a product on publicly available websites and blogs, automated notifications of users' activities and facilitation of personalized invitations. The latter are more common and are investigated in this study.

Personalized referrals/invitations /// Personalized referral features allow users to select their friends or contacts from a list and invite them to adopt a product or service, with the option of attaching a personalized message to the invitation. Social networking websites enable users to "invite their friends" to join the service through personalized referrals. When users send an email from a Web-based service, for example Gmail, an automated pop-up hyperlink prompts them to invite the recipients to join the service.

Automated broadcast notifications /// Automated broadcast notifications are automatically triggered by normal user activity. When users engage with the product in a certain way (e.g., send a message, update their status), those actions are broadcast as notifications to their contact list. Notifications build awareness among friends of new activities or products that users are adopting or engaging with. This information can encourage those friends to eventually adopt the product themselves. For example, social networking websites typically post a notification automatically when a user adopts a new app or achieves some app milestone. 


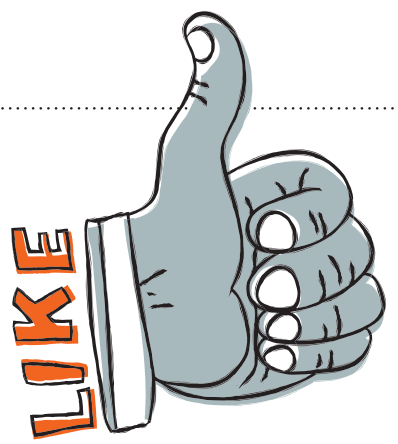

To analyze the success of these viral features, we collaborated with a Facebook developer and a client to develop three versions of a free app that provides users the opportunity to share information and opinions about movies, actors, directors and the film industry in general.

One group received the app without any viral features, the second group received the app with a feature to send personalized invitations, and for the third group, passivebroadcast notifications were enabled. All three versions also collected personal attributes and preferences from users' Facebook profiles, as well as data on their social networks and the personal attributes and preferences of their network neighbors.

By comparing peer adoption across the randomly selected users of each group, researchers were able to observe the impact of the viral features. Using detailed clickstream data on users' online behaviors, they were further able to estimate adoption rates beyond the immediate network of original users and to analyze further product adoption and sustained product use.

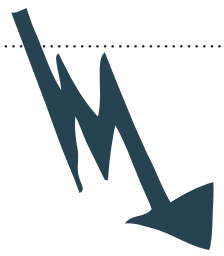

EXPERIMENT OVERVIEW

\section{9,687 INITIAL USERS}
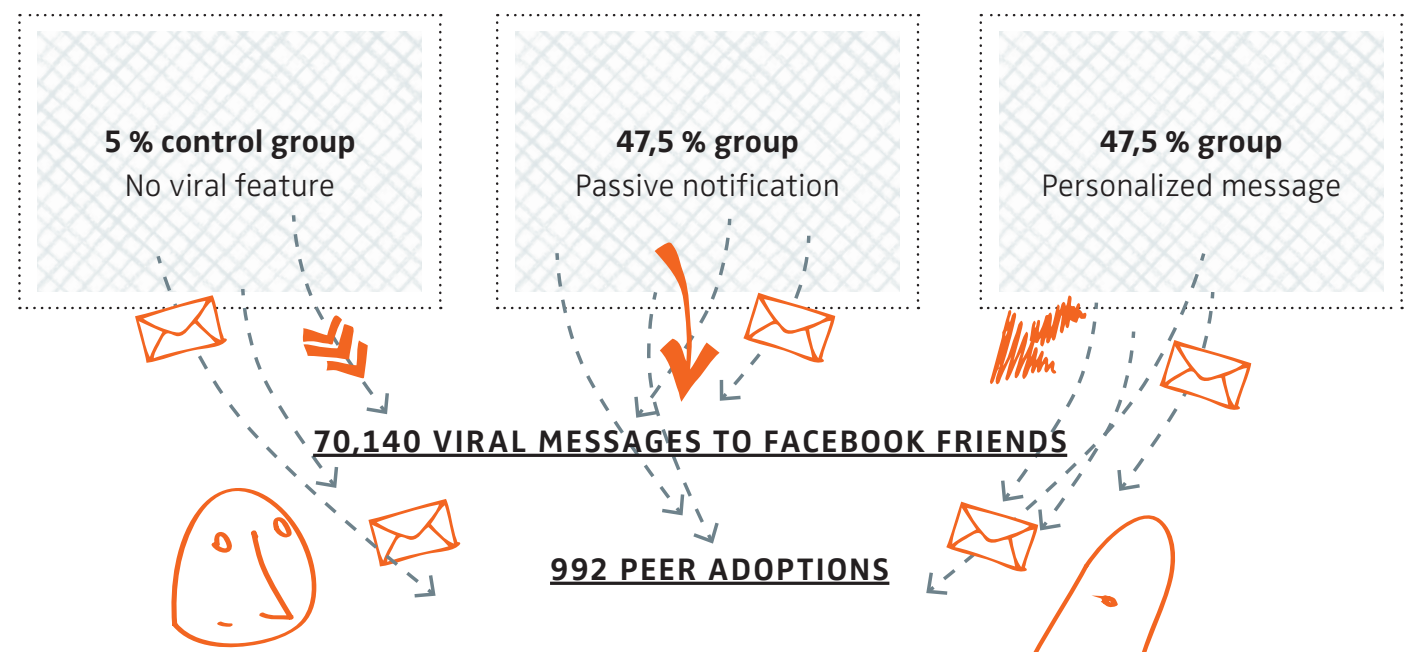

AGES TO FACEBOOK FRIEND'́
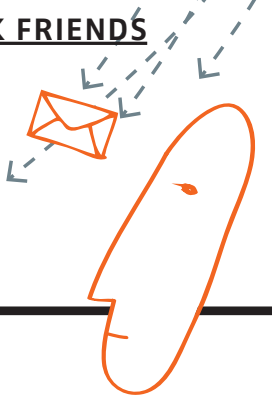

992 PEER ADOPTIONS 


\section{OH!}

FIGURE 1:

Viral features increase adoption, speed of diffusion and use of the app

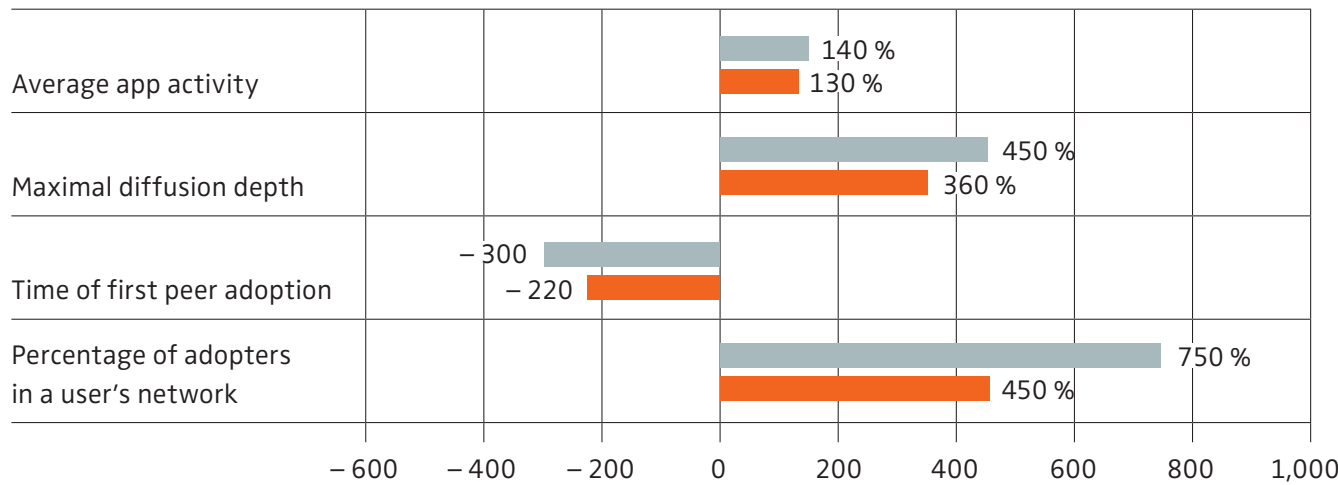

\section{$\gg$ \\ Compared with classic \\ online advertising, invitations \\ or notifications \\ seem to be a good alternative.}

《<

\section{Notifications created more adoption but less engage- ment and lower yield per message /// In online environ- ments in which consumers are bombarded with irrelevant information, more personalized-active features, such as invi- tations, had a greater yield - in this case the adoption rate per message - than passive-broadcast features.}

Although each personalized referral had a much stronger impact, notifications outperformed the invitations in overall adoption. Figure 2 shows the results of a clickstream analy- sis and illustrates the effect. Notifications require no user effort and are sent automatically to peers. Therefore, substantially more messages were generated. Personalized features require more initiative and activity from the user. The number of users who made this effort was much smaller. Overall, users of the passive-broadcast app experienced a $246 \%$ increase in the rate of app adoption, whereas adding active-personalized viral messaging capabilities generated only an additional $98 \%$ increase in adoption compared with the group without viral features. However, personal invitations created more engagement among users than broadcast notifications, increasing the intensity of use and reducing churn.

\section{Insights for managers}

The results suggest that it pays off to integrate viral features into products. Compared with classic online advertising, invitations or notifications seem to be a good alternative - at least as long as their use is limited. Bombarding users with messages from peers may reduce their effectiveness and the overall quality of the user experience, but a comparison of success rates at the time of the experiment was impressive. 
FIGURE 2:

\section{Overall increase in the rate of adoption}

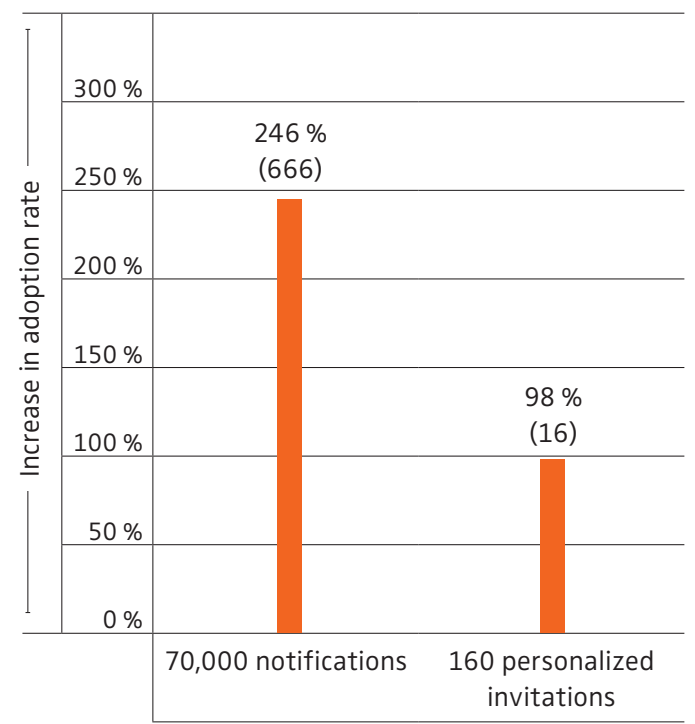

Adoption rate:

Invitations: $0.1 \%$, Notifications: $0.01 \%$
Viral features outperform typical Facebook ad and paid search campaigns /// The tested app spread faster and further with either viral feature and seemed more effective in encouraging new product adoption than traditional marketing campaigns. Notifications produced a $1 \%$ conversion rate, and invitations had a $10 \%$ conversion rate. Both vastly outperformed the Facebook ad campaign used in the recruitment phase of the experiment, which produced only a $0.01 \%$ conversion rate. Because Facebook currently has the largest market share of display advertising on the Web, these comparisons reflect the relative performance of viral product design and the lion's share of Web-based display advertising. The personalized invitations also outperformed typical conversion rates in paid search advertising, which are estimated at $0.02 \%$.
Viral features outperform typical click-through rates /// Although conversion rates are typically smaller than clickthrough rates, the viral product features outperformed in this measure as well. The $1 \%$ conversion rate on notifications is higher than the typical click-through rates of banner advertising, which usually range from $0.1 \%$ to $0.2 \%$. Invitations with a conversion rate of $10 \%$ are more effective than email marketing campaigns with click-through rates ranging from $2 \%$ to $6 \%$.

Implementation of viral features is fairly inexpensive /// The implementation of viral features incurred a low, onetime fixed cost of approximately $\$ 600$. The expected return, on the other hand, was proportional to the increase in adopters the feature generated. Viral product design may be more cost-effective than increased spending on traditional digital advertising, which incurs costs proportional to impressions or clicks. To reach an initial base of users, however, traditional advertising might be necessary.

\section{Optimizing viral features might increase effectiveness}

/// Active-personalized features work well, but the large amount of effort required of users curtails their use. One solution might be to couple active-personalized features with referral incentives that encourage use. Optimally designed incentive strategies could motivate users to generate more personalized referrals and to target and personalize viral messages more effectively.

\section{Reinforce feedback loops on product adoption ///} Another option might be to integrate feedback capabilities into the viral features. The app could generate automated notifications when a peer adopts or uses the app in a certain way. Results show that additional users among one's friends drive a positive feedback loop: Product use drives peer adoption, and peer adoption in turn drives product use. Managers could try to reinforce this feedback loop by designing viral features and feedback tools into their products.

\section{Managerial summary of an article published in the academic top journal "Management Science":}

Aral, Sinan; Walker, Dylan (2011): "Creating Social Contagion through Viral Product Design: A Randomized Trial of Peer Influence in Networks", Management Science, (September), pp. $1623-1639$. 